\title{
El control estricto de la glucemia también beneficia al diabético tipo II
}

The Effects of Improved Glycemic Control on Complications in Type 2 Diabetes. Barak Gaster; Irl B. Hirsch. Arch Intern Med 1998;158:134-40.

\section{Objetivo}

Evaluar la evidencia científica que relaciona el control de la glucemia con la incidencia y la progresión de las complicaciones crónicas de la diabetes.

\section{Fuentes de datos}

Se obtuvieron por MEDLINE todos los estudios en inglés desde 1970 en adelante, que investigaran el nivel de control de la glucemia y la presencia de complicaciones.

\section{Selección de estudios}

Se incluyeron estudios de cohortes prospectivas y ensayos clínicos controlados y aleatorizados de más de tres meses de seguimiento. Se excluyeron aquellos que no diferenciaron el tipo de diabetes (I ó II), los diabéticos tipo I de II, los que no usaron adecuadas medidas de control (glucemia en ayunas o hemoglobina glicosilada) y los que no reportaron análisis estadístico.

\section{Resultados principales}

os estudios epidemiológicos mostraron una fuerte asociación entre la hiperglucemia y la tasa de complicaciones microvasculares (20 estudios analizables). La relación fue la misma para los tipo I que para los tipo II para cualquier nivel dado de hiperglucemia.

Proteínuria y retinopatía: de los tres ensayos clínicos randomizados y controlados publicados, el UGDP (University Group Diabetes Program ) no mostró diferencias luego de 13 años de sequimiento. El estudio japonés (Kumamoto) mostró una reducción del 69\% (IC 95\% 24-87\%) en la incidencia de retinopatía en el grupo de tratamiento convencional vs. el agresivo (de 38\% a 13\%, p < 0,01), 70\% de reducción (IC 95\% 14\% - $89 \%$ ) de nefropatía (de $30 \%$ a $10 \%, p<0,005$ ) y reducción de neuropatía (de 64,6 a $12,8 \%$ de aumento en el umbral de vibración de las extremidades inferiores $(p<0,05)$. El estudio de los veteranos mostró una disminución significativa de la hemoglobina glicosilada en el grupo de tratamiento intensivo vs. el convencional al final de 27 meses de seguimiento y una disminución de la aparición y progresión de microalbuminuria, pero no observó disminución de la retinopatía.

Enfermedad macrovascular: dos estudios mostraron disminución de los eventos de la mortalidad cardiovascular con el tratamiento intensivo vs. el habitual pero uno de ellos (VA CSDM) no logró significancia estadística. Las dificultades que presentó el control estricto fueron: hipoglucemia $(0,03$ episodios de hipoglucemia por paciente por año en el grupo de

Fuente de financiamiento: No referida tratamiento intensivo vs 0,01 en el standard), ganancia de peso y alteración de la calidad de vida.

Pacientes en los estudios analizados con complicaciones macro y microvasculares como puntos finales

\begin{tabular}{|c|c|c|c|c|c|c|c|}
\hline Estudio & $\begin{array}{l}\text { Tipo de } \\
\text { diabetes }\end{array}$ & $\begin{array}{l}\mathrm{N}^{\circ} \text { de } \\
\text { pacientes }\end{array}$ & $\begin{array}{l}\text { Duración } \\
\text { en años }\end{array}$ & $\begin{array}{l}\text { Puntos } \\
\text { finales }\end{array}$ & $\begin{array}{l}\text { Control } \\
\text { estricto }\end{array}$ & $\begin{array}{l}\text { Control } \\
\text { usual }\end{array}$ & $p$ \\
\hline DIGAMI & $\begin{array}{l}85 \% 2, \\
15 \% 1\end{array}$ & 620 & 1,0 & Mortalidad & $18,6 \%$ & $26,1 \%$ & 0,03 \\
\hline DCCT & 1 & 1441 & 6,5 & Eventos EC & $0,06 \%$ año & 0,29\%año & 0,06 \\
\hline WESDR & $1 y^{2}$ & $\begin{array}{l}1780 \\
\text { (tipo2) }\end{array}$ & 10 & $\begin{array}{l}\text { R, P, Ny } \\
\text { Hbgili }\end{array}$ & Insulina & NoInsulina & $\begin{array}{l}\text { compli- } \\
\text { caciones } \\
\text { micro }\end{array}$ \\
\hline \multirow[t]{2}{*}{ UGDP } & 2 & 619 & 12,5 & IAM & $20,6 \%$ & $20,2 \%$ & 1,00 \\
\hline & & & & RyP & $\begin{array}{l}\text { Insulina, } \\
\text { dosis } \\
\text { variable }\end{array}$ & $\begin{array}{l}\text { Insulina, } \\
\text { dosis fijas } \\
\text { y placebo }\end{array}$ & $\begin{array}{l}\text { No dife- } \\
\text { rencias } \\
\text { signif. }\end{array}$ \\
\hline \multirow[t]{2}{*}{ Kumamoto } & & 110 & 6,0 & $\begin{array}{l}\text { IAM o } \\
\text { angina }\end{array}$ & $n=1$ & $n=1$ & \\
\hline & & & & $\begin{array}{l}\mathrm{R} \\
\mathrm{P} \\
\mathrm{N} \\
\mathrm{Hb} \text { gli }\end{array}$ & $\begin{array}{l}13 \% \\
10 \% \\
12,8 \% \\
7,1 \%\end{array}$ & $\begin{array}{l}38 \% \\
30 \% \\
64,6 \% \\
9,4 \%\end{array}$ & $\begin{array}{l}0,007 \\
0,005 \\
0,05\end{array}$ \\
\hline \multirow[t]{4}{*}{ VA CSDM } & 2 & 153 & 2,3 & Eventos EC & $21,3 \%$ & $11,5 \%$ & 0,10 \\
\hline & & & & $P$ & $\begin{array}{l}14 \text { a } 158 \\
\mathrm{mg} / 24 \mathrm{hs} \\
(\mathrm{p:} 0,008)\end{array}$ & $\begin{array}{l}11 \text { a } 44 \\
\mathrm{mg} / 24 \mathrm{hs}\end{array}$ & $\begin{array}{l}\text { no se } \\
\text { hicieron }\end{array}$ \\
\hline & & & & $R$ & & & $\begin{array}{l}\text { no dife- } \\
\text { rencias }\end{array}$ \\
\hline & & & & $\mathrm{Hbgli}$ & $7,3 \%$ & $9,4 \%$ & 0,001 \\
\hline
\end{tabular}

UGDP: University Group Diabetes Program; VA CSDM: Veterans Affairs Cooperative Study on Glycemic Control and Complications in Type II Diabetes; Kumamoto: Study conducted in Kumanoto, japón: DIGAMI: Diabetes and Insulin in Acute Myocardial Infarction; DCCT: Diabetes Control and Complication Trial. WESDR: Wisconsin Epidemiologic Study of Diabetic Retinopathy; R: retinopatía, P: proteinuria o nefropatía, N: neuropatía; Hb gli: hemoglobina glicosilada. Eventos EC: eventos coronanos.

\section{Conclusiones}

Hay evidencia que avala la mejoría del control glucémico. Previene o retarda la retino, nefro y neuropatía diabéticas en los tipo II. No hay evidencia contundente que el control estricto empeore la enfermedad coronaria en este grupo; y la hipoglucemia es 10 a 100 veces menos común que en los tipo I. La hemoglobina glicosilada debería mantenerse entre 7 y $8 \mathrm{gr} \%$.

\section{COMENTARIO}

Con la publicación del DCCT (1) se demostró claramente que el control estricto de la glucemia reducía la incidencia y progresión de complicaciones de la diabetes en pacientes tipo I. Si éstos resultados fueran transferibles a los pacientes con diabetes tipo II se modificaría el seguimiento y el tratamiento del $80 \%$ de los diabéticos, que en su mayoría son atendidos por los médicos de atención primaria. El impacto sería enorme, ya que deberíamos ofrecerle a todos los diabéticos que atendemós habitualmente el conjunto escalonado de terapias para normalizar sus glucemias con tiempos no muy prolongados entre uno y otro tratamiento. Algunas revisiones previas (2) señalaban como más peligroso la posibilidad de daño aterosclerótico por hiperinsulinemia (exógena).

Esta revisión es muy útil ya que resume y agrupa la evidencia actual: 1. Existe una tendencia clara a la disminución de las complicaciones microvasculares con el control más estricto de la glucemia en los diabéticos tipo 2 que se manifiesta luego de por lo menos 2 años del inicio del tratamiento intensivo (ésta puede ser la razón por la cual no se observaron diferencias en el VACSDM)

El estudio japonés (4), utilizó un diseño muy similar al DCCT y reportó beneficios similares en diabéticos tipo 2. De todas maneras, la validez externa de ese estudio se ve limitada por las características étnicas y clíni- cas de los participantes, ya que no representan a los diabéticos tipo 2 vistos típicamente en nuestra práctica.

2. No hay convincente evidencia de que el control estricto empeore la enfermedad coronaria. Aquí reside la principal incertidumbre ya que los únicos dos trabajos que muestran diferencias en la cantidad de eventos fueron el DCCT en diabéticos tipo I y el DIGAMI en población diabética infartada, cuadros clínicos que probablemente se alejen de la situación clínica habitual de nuestros diabéticos tipo II seguidos en forma ambulatoria. 3. Es necesario aquardar los resultados de estudios clínicos aleatorizados en diabéticos tipo II. Mientras tanto debemos, como siempre, aplicar el juicio clínico en el paciente individual, teniendo en cuenta sus preferencias y motivaciónes.

Factores como edad menor de 60 años, otros factores de riesgo coronario, historia familiar de nefropatía, retinopatía temprana y microalbuminuria inclinan claramente la balanza hacia el control estricto. Por el contrario, factores como edad mayor de 70 años, complicaciones diabéticas avanzadas, incapacidad de reconocer una hipoglucemia, enfermedad coronaria avanzada, demencia y falta de motivación disminuyen el beneficio potencial del control estricto y aumentan la posibilidad de efectos adversos.

Genuth S. Exogenous insulin administration and cardiovascular risk in non-insulin-dependent and insulin-dependent diabetes mellitus. Ann Intern Med.1996; 124: 104-109.

3. American Diabetes Association. Standards of medical care for patients with diabetes mellitus. Diabetes Care. 1996; 19(suppl): S8-15.

4. Ohkubo Y, Kishikawa H, Araki E et al. Intensive insulin therapy prevents the progression of microvascular complications in Japanese patients with non insulin dependent diabetes mellitus: 